PROCEEDINGS OF THE

AMERICAN MATHEMATICAL SOCIETY

Volume 125, Number 6, June 1997, Pages 1579-1583

S 0002-9939(97)03704-0

\title{
UPPER BOUNDS FOR THE NUMBER OF FACETS OF A SIMPLICIAL COMPLEX
}

\author{
JÜRGEN HERZOG AND TAKAYUKI HIBI
}

(Communicated by Wolmer V. Vasconcelos)

\begin{abstract}
Here we study the maximal dimension of the annihilator ideals $0: A m^{j}$ of artinian graded rings $A=P /\left(I, x_{1}^{2}, x_{2}^{2}, \ldots, x_{v}^{2}\right)$ with a given Hilbert function, where $P$ is the polynomial ring in the variables $x_{1}, x_{2}, \ldots, x_{v}$ over a field $K$ with each $\operatorname{deg} x_{i}=1, I$ is a graded ideal of $P$, and $m$ is the graded maximal ideal of $A$. As an application to combinatorics, we introduce the notion of $j$-facets and obtain some informations on the number of $j$-facets of simplicial complexes with a given $f$-vector.
\end{abstract}

Let $P=K\left[x_{1}, x_{2}, \ldots, x_{v}\right]$ denote the polynomial ring in $v$ variables over a field $K$ with the standard grading, i.e., each $\operatorname{deg} x_{i}=1$, and write $K\{\Gamma\}$ for the quotient algebra $P /\left(x_{1}^{2}, x_{2}^{2}, \ldots, x_{v}^{2}\right)$. We are interested in the dimensions of the annihilator ideals $0: K\{\Gamma\} / I m^{j}$ of $K\{\Gamma\} / I$, where $m$ is the graded maximal ideal of $K\{\Gamma\} / I$. In particular, among all graded ideals $I$ of $K\{\Gamma\}$ with a given Hilbert function, we determine the maximal dimension of the socles $0:_{K\{\Gamma\} / I} m$ of $K\{\Gamma\} / I$. The graded ring $K\{\Gamma\} / I$ is studied in $[\mathrm{A}-\mathrm{H}-\mathrm{H}]$ when $I$ is generated by (squarefree) monomials.

First, we recall some standard notation and terminology on graded rings and modules. When $M$ is a $\mathbf{Z}$-graded module, where $\mathbf{Z}$ is the set of integers, we write $M_{i}, i \in \mathbf{Z}$, for the $i$-th graded component of $M$. Moreover, for every $a \in \mathbf{Z}$, we define $M(a)$ to be the $\mathbf{Z}$-graded module with graded components $M(a)_{i}=M_{a+i}$ for all $i \in \mathbf{Z}$. If $M$ is a finitely generated $\mathbf{Z}$-graded module over the polynomial ring $P=K\left[x_{1}, x_{2}, \ldots, x_{v}\right]$, then the modules $\operatorname{Tor}_{i}^{K}(K, M)$ are finite-dimensional graded $K$-vector spaces. Then we say that $\beta_{i j}(M):=\operatorname{dim}_{K} \operatorname{Tor}_{i}^{K}(K, M)_{j}$ is the $(i, j)$-th graded Betti number of $M$. Finally, when $A$ is a graded ring over $K$ and $J$ is a graded ideal of $A$, we denote by $0:{ }_{A} J$ the annihilator of $J$ in $A$.

Let $\left(\begin{array}{l}V \\ q\end{array}\right)$ denote the set of all squarefree monomials of degree $q \geq 1$ in the variables $V=\left\{x_{1}, x_{2}, \ldots, x_{v}\right\}$. We write $\leq_{\text {lex }}$ for the lexicographic order on $\left(\begin{array}{l}V \\ q\end{array}\right)$, i.e., if $S=x_{i_{1}} x_{i_{2}} \cdots x_{i_{q}}$ and $T=x_{j_{1}} x_{j_{2}} \cdots x_{j_{q}}$ are squarefree monomials belonging to $\left(\begin{array}{l}V \\ q\end{array}\right)$ with $1 \leq i_{1}<i_{2}<\cdots<i_{q} \leq v$ and $1 \leq j_{1}<j_{2}<\cdots<j_{q} \leq v$, then $S<_{\text {lex }} T$ if $i_{1}=j_{1}, \ldots, i_{k-1}=j_{k-1}$ and $i_{k}>j_{k}$ for some $1 \leq k \leq q$. A nonempty set $\mathcal{M} \subset\left(\begin{array}{l}V \\ q\end{array}\right)$ is called a squarefree lexsegment set of degree $q$ if $T \in \mathcal{M}, S \in\left(\begin{array}{l}V \\ q\end{array}\right)$ and $T \leq_{\text {lex }} S$ imply $S \in \mathcal{M}$. An ideal $I$ of $K\{\Gamma\}$ generated by squarefree monomials is

Received by the editors August 28, 1995 and, in revised form, October 26, 1995.

1991 Mathematics Subject Classification. Primary 05D05; Secondary 13D40.

This paper was written while the authors were staying at the Mathematische Forschungsinstitut Oberwolfach in the frame of the RiP program which is financed by Volkswagen-Stiftung.

(C)1997 American Mathematical Society 
called a squarefree lexsegment ideal if, for every $1 \leq q \leq v, T \in I \cap\left(\begin{array}{l}V \\ q\end{array}\right), S \in\left(\begin{array}{l}V \\ q\end{array}\right)$ and $T<_{\text {lex }} S$ imply $S \in I$.

We are now in the position to state our algebraic result of this paper.

Theorem. (a) Suppose that I is a graded ideal of $K\{\Gamma\}$ with $I_{0}=I_{1}=(0)$. Then, there exists a unique squarefree lexsegment ideal $I^{\text {lex }}$ of $K\{\Gamma\}$ with the same Hilbert function as $I$.

(b) Let $m$ be the graded maximal ideal of $K\{\Gamma\}$. Fix $j \geq 0$. Suppose that for every $i \geq 0$ we have

$$
\operatorname{dim}_{K}\left(I / m^{j} I\right)_{i}=\operatorname{dim}_{K}\left(I^{\text {lex }} / m^{j} I^{\text {lex }}\right)_{i} .
$$

Then for every $i \geq 0$ we have

$$
\operatorname{dim}_{K}\left(I / m^{j+1} I\right)_{i} \leq \operatorname{dim}_{K}\left(I^{\mathrm{lex}} / m^{j+1} I^{\mathrm{lex}}\right)_{i} .
$$

(c) Let $A=K\{\Gamma\} / I$ and $B=K\{\Gamma\} / I^{\text {lex }}$. Fix $j \geq 0$. Suppose that for every $i \geq 0$ we have

$$
\operatorname{dim}_{K}\left(0:_{A} m^{j}\right)_{i}=\operatorname{dim}_{K}\left(0:_{B} m^{j}\right)_{i}
$$

Then for every $i \geq 0$ we have

$$
\operatorname{dim}_{K}\left(0:_{A} m^{j+1}\right)_{i} \leq \operatorname{dim}_{K}\left(0:_{B} m^{j+1}\right)_{i} .
$$

Proof. First, we choose any term order $\rho$ for the monomials in the polynomial ring $P=K\left[x_{1}, x_{2}, \ldots, x_{v}\right]$ and we write $J \subset P$ for the preimage of $I$ under the canonical epimorphism $P \rightarrow K\{\Gamma\}$. It is well known (e.g., [M-M], [B-H-V]) that $P / J$ and $P / \operatorname{in}_{\rho}(J)$ have the same Hilbert function and that we have the inequality $\beta_{i j}(P / J) \leq \beta_{i j}\left(P / \operatorname{in}_{\rho}(J)\right)$ for every $i$ and $j$.

We have the equalities $\beta_{1 j}(P / J)=\operatorname{dim}_{K}(I / m I)_{j}$ if $j>2 ; \beta_{12}(P / J)=$ $\operatorname{dim}_{K}(I / m I)_{2}-v$; and $\beta_{v j}(P / J)=\operatorname{dim}_{K} \operatorname{Soc}_{j-v}(P / J)$ for every $j>v$. The similar results hold for the ideal $\operatorname{in}_{\rho}(J) \subset P$ and its image $I^{\prime}$ in $K\{\Gamma\}$. Since $K\{\Gamma\} / I \simeq P / J$ and $K\{\Gamma\} / I^{\prime} \simeq P / \operatorname{in}_{\rho}(J)$, it follows that $K\{\Gamma\} / I$ and $K\{\Gamma\} / I^{\prime}$ have the same Hilbert function, and that $\operatorname{dim}_{K}(I / m I)_{i} \leq \operatorname{dim}_{k}\left(I^{\prime} / m I^{\prime}\right)_{i}$ and $\operatorname{dim}_{K} \operatorname{Soc}_{i}(K\{\Gamma\} / I) \leq \operatorname{dim}_{K} \operatorname{Soc}_{i}\left(K\{\Gamma\} / I^{\prime}\right)$ for every $i$.

Thus, replacing $I$ with $I^{\prime}$ and noting that $I^{\prime}$ is generated by squarefree monomials, we may assume from the beginning that $I$ itself is generated by squarefree monomials.

Now since $I$ is an ideal in $K\{\Gamma\}$ generated by squarefree monomials, the existence (and uniqueness) of $I^{\text {lex }}$ is an immediate consequence of the Kruskal-Katona theorem which, stated in algebraic language, guarantees the following: Suppose that $L \subset K\{\Gamma\}$ is an ideal generated by squarefree monomials, all of the same degree, say $q$, and let $L^{\text {lex }}$ denote the ideal generated by the squarefree lexsegment set $\mathcal{M}$ of degree $q$ with $\sharp(\mathcal{M})=\operatorname{dim}_{K} L_{q}$. Then $\operatorname{dim}_{K} L_{q+1} \geq \operatorname{dim}_{K}\left(L^{\text {lex }}\right)_{q+1}$. Thanks to this fact, given a squarefree ideal $I$ of $K\{\Gamma\}$, if we consider for each $i$ the vector space $V_{i} \subset K\{\Gamma\}$ spanned by the squarefree lexsegment set $\mathcal{M}_{i}$ of degree $i$ with $\sharp\left(\mathcal{M}_{i}\right)=\operatorname{dim}_{K} I_{i}$, then $\bigoplus_{i \geq 0} V_{i}$ is an ideal of $K\{\Gamma\}$, which is just the desired $I^{\text {lex }}$. This construction also enables us to see that in each degree the number of generators of $I^{\text {lex }}$ is greater than or equal to that of $I$, which proves the inequalities in (b) for $j=0$.

Now suppose that $j>0$. Our hypothesis implies that $m^{j} I$ and $m^{j} I^{\text {lex }}$ have the same Hilbert function. Therefore, since $m^{j} I^{\text {lex }}$ is a lexsegment ideal, we conclude that $\left(m^{j} I\right)^{\mathrm{lex}}=m^{j} I^{\mathrm{lex}}$. Thus, as above, we deduce from the KruskalKatona theorem that in each degree the number of generators of $m^{j} I^{\text {lex }}$ is greater 
than or equal to that of $m^{j} I$. In other words, we have $\operatorname{dim}_{K}\left(m^{j} I / m^{j+1} I\right) \leq$ $\operatorname{dim}_{K}\left(m^{j} I^{\text {lex }} / m^{j+1} I^{\text {lex }}\right)$. This completes the proof of the inequalities in (b) as desired.

The inequalities (c) will turn out to be again a consequence of the KruskalKatona theorem, but not quite as straightforward. Let us first consider the canonical module $\omega_{A}$ of $A=K\{\Gamma\} / I$. We refer the reader to, e.g., [B-H] for basic facts about canonical modules. Since $K\{\Gamma\}$ is a Gorenstein ring (in fact, a complete intersection), we may represent $\omega_{A}$, up to a shift, as a module of homomorphisms, that is to say, we have $\omega_{A}(-v)=\operatorname{Hom}_{K\{\Gamma\}}(A, K\{\Gamma\})$. The module $\operatorname{Hom}_{K\{\Gamma\}}(A, K\{\Gamma\})$ of homomorphisms may be naturally identified with the annihilator of $I$ in $K\{\Gamma\}$. Hence, as a graded module, $\omega_{A}(-v)$ may be regarded as the ideal in $K\{\Gamma\}$ whose $K$-basis $\Omega$ is given by all squarefree monomials $T \in K\{\Gamma\}$ which annihilate $I$.

We claim that $\Omega$ is the set of all squarefree monomials $T^{c} \in K\{\Gamma\}$ such that $T \notin I$, where $T^{c}$ is defined by $T^{c}=x_{1} \cdots x_{v} / T$. In fact, suppose that $T \notin I$ and that $T^{c} S \neq 0$ for some squarefree monomial $S \in I$. Then, there exists a squarefree monomial $U$ such that $T^{c} S U=x_{1} \cdots x_{v}=T T^{c}$. Hence $T=S U$, and thus $T \in I$, a contradiction. Conversely, since $T T^{c}=x_{1} \cdots x_{v} \neq 0$, if $T^{c} I=0$ then $T \notin I$ as desired.

It follows from the identification of $\omega_{A}(-v)$ with the annihilator of $I$ that $\operatorname{dim}_{K}\left(\omega_{A}\right)_{i}=\operatorname{dim}_{K} A_{-i}$ for every $i$. Thus, in particular, if $B=K\{\Gamma\} / I^{\text {lex }}$, then the ideals $\omega_{A}(-v)$ and $\omega_{B}(-v)$ have the same Hilbert function.

The reader can verify easily, by the above description of the canonical module, that $\omega_{B}(-v)$ is a squarefree lexsegment ideal of $K\{\Gamma\}$. In fact, if $\mathcal{M}$ is a squarefree lexsegment set of degree $q$ and if $\mathcal{K}$ is the complement of $\mathcal{M}$ in the set of all squarefree monomials of degree $q$, then the set $\left\{T^{c} ; T \in \mathcal{K}\right\}$ is again a squarefree lexsegment set of degree $v-q$. This observation is crucial, since it implies that $\omega_{B}(-v)=\left(\omega_{A}(-v)\right)^{\text {lex }}$.

Finally we notice that, for every finite dimensional graded $K$-algebra $C$ and for all integers $i, j \geq 0$, we have $\operatorname{dim}_{K}\left(0:_{C} m^{j}\right)_{i}=\operatorname{dim}_{K}\left(\omega_{C} / m^{j} \omega_{C}\right)_{-i}$. Thus if we apply the arguments in the proof of (b) to the ideal $\omega_{A}(-v)$, then the assertion (c) follows.

Q. E. D.

By virtue of the Clements-Lindström theorem $[\mathrm{C}-\mathrm{L}]$, the above Theorem can be generalized to ideals of the quotient algebra $P /\left(x_{1}^{a_{1}}, x_{2}^{a_{2}}, \ldots, x_{v}^{a_{v}}\right)$ with $1 \leq a_{1} \leq$ $a_{2} \leq \cdots \leq a_{v}$.

We now discuss the combinatorial implication of the above result. Let $\Delta$ be a simplicial complex on the vertex set $V=\left\{x_{1}, x_{2}, \ldots, x_{v}\right\}$, i.e., $\Delta$ is a collection of subsets of $V$ such that (i) $\left\{x_{i}\right\} \in \Delta$ for every $1 \leq i \leq v$ and (ii) if $\sigma \in \Delta$ and $\tau \subset \sigma$ then $\tau \in \Delta$. Each element $\sigma$ of $\Delta$ is called a face of $\Delta$. A facet of $\Delta$ is a face $\sigma$ of $\Delta$ such that $\tau \in \Delta$ and $\sigma \subset \tau$ imply $\sigma=\tau$. Let $f_{i}=f_{i}(\Delta)$ be the number of faces $\sigma$ of $\Delta$ with $\sharp(\sigma)=i+1$, and $n_{i}=n_{i}(\Delta)$ the number of facets $\sigma$ of $\Delta$ with $\sharp(\sigma)=i+1$. Here, $\sharp(\sigma)$ is the cardinality of a finite set $\sigma$. Note that $f_{-1}=1$ and $n_{-1}=0$. We say that $f(\Delta)=\left(f_{0}, f_{1}, \ldots\right)$ is the $f$-vector of $\Delta$. Let $P=K\left[x_{1}, x_{2}, \ldots, x_{v}\right]$ denote the polynomial ring in $v$ variables over a field $K$ as before, and define $I_{\Delta}$ to be the ideal of $P$ generated by all squarefree monomials $x_{i_{1}} x_{i_{2}} \cdots x_{i_{r}}, 1 \leq i_{1}<i_{2}<\cdots<i_{r} \leq v$, with $\left\{x_{i_{1}}, x_{i_{2}}, \ldots, x_{i_{r}}\right\} \notin \Delta$. The quotient algebra $P / I_{\Delta}$ is called the Stanley-Reisner ring of $\Delta$ over $K$. We refer the reader to, e.g., $[\mathrm{B}-\mathrm{H}],[\mathrm{H}],[\mathrm{Hoc}]$ and $[\mathrm{Sta}]$ for detailed information about Stanley-Reisner rings. 
Now, we write $I_{\Delta}^{\prime}$ for the image of $I_{\Delta}$ in $K\{\Gamma\}$ and set $K\{\Delta\}=K\{\Gamma\} / I_{\Delta}^{\prime}$. Then, the Hilbert function of $K\{\Delta\}$ coincide with the $f$-vector of $\Delta$, i.e., $\operatorname{dim}_{K}(K\{\Delta\})_{i}=$ $f_{i-1}(\Delta)$ for each $i \geq 0$. A simplicial complex $\Delta$ is called lexsegment if the ideal $I_{\Delta}^{\prime}$ of $K\{\Gamma\}$ is a squarefree lexsegment ideal. Let $\Delta^{\text {lex }}$ denote the unique lexsegment simplicial complex with the same $f$-vector as $\Delta$. Then, $\left(I_{\Delta}^{\prime}\right)^{\text {lex }}=I_{\Delta^{\text {lex }}}^{\prime}$.

Recall from, e.g., $[\mathrm{H}]$ or $[\mathrm{B}-\mathrm{H}]$ that, given positive integers $f$ and $i$, there exists a unique representation of $f$ of the form

$$
\begin{gathered}
f=\left(\begin{array}{c}
a_{i} \\
i
\end{array}\right)+\left(\begin{array}{c}
a_{i-1} \\
i-1
\end{array}\right)+\cdots+\left(\begin{array}{c}
a_{j} \\
j
\end{array}\right), \\
a_{i}>a_{i-1}>\cdots>a_{j} \geq j \geq 1 .
\end{gathered}
$$

We then define

$$
\partial_{i-1}(f)=\left(\begin{array}{c}
a_{i} \\
i-1
\end{array}\right)+\left(\begin{array}{c}
a_{i-1} \\
i-2
\end{array}\right)+\cdots+\left(\begin{array}{c}
a_{j} \\
j-1
\end{array}\right) .
$$

Also, we set $\partial_{i-1}(0)=0$ for every $i \geq 1$. It is an excercise in combinatorics (see, e.g., $[\mathrm{G}-\mathrm{K}])$ to show that, if $\Delta$ is a lexsegment simplicial complex with $f(\Delta)=$ $\left(f_{0}, f_{1}, \ldots\right)$, then $\partial_{i}\left(f_{i}\right) \leq f_{i-1}$ and $n_{i-1}=f_{i-1}-\partial_{i}\left(f_{i}\right)$ for each $i \geq 1$.

Corollary. Let $\Delta$ be a simplicial complex with $f$-vector $\left(f_{0}, f_{1}, \ldots\right)$. Then, for each $i \geq 1$, we have the inequality $n_{i-1} \leq f_{i-1}-\partial_{i}\left(f_{i}\right)$. Moreover, when $\Delta$ is lexsegment, the equality $n_{i-1}=f_{i-1}-\partial_{i}\left(f_{i}\right)$ holds for every $i \geq 1$.

Proof. Since $\operatorname{dim}_{K}\left(0:_{K\{\Delta\}} m\right)_{i}=n_{i-1}(\Delta)$, the inequality with $j=0$ in (c) of the theorem is equal to the required inequality $n_{i-1}(\Delta) \leq n_{i-1}\left(\Delta^{\text {lex }}\right)$ for every $i \geq 0$.

Q. E. D.

The above inequalities are a part of a more general conjecture in $[\mathrm{A}-\mathrm{H}-\mathrm{H}]$ on graded Betti numbers of ideals of the form $\left(I_{\Delta}, x_{1}^{2}, x_{2}^{2}, \ldots, x_{v}^{2}\right)$.

It would, of course, be of interest to find all possible sequences $\left(n_{0}, n_{1}, \ldots\right)$ arising from the simplicial complexes with a given $f$-vector.

We now introduce the concept of $j$-facets of simplicial complexes. We say that a face $\sigma$ of a simplicial complex $\Delta$ is a $j$-facet if $j$ is equal to the greatest integer $k \geq 0$ for which there exists a face $\tau$ of $\Delta$ such that $\sigma \cap \tau=\emptyset, \sigma \cup \tau \in \Delta$ and $\sharp(\tau)=k$. Thus, in particular, the 0 -facets of $\Delta$ are just the facets of $\Delta$. Let $n_{i}^{j}=n_{i}^{j}(\Delta)$ denote the number of $j$-facets $\sigma$ of $\Delta$ with $\sharp(\sigma)=i+1$. For example, if $\Delta$ is a simplicial complex with $f(\Delta)=(5,7,1)$, then $n_{i}^{j}(\Delta)=n_{i}^{j}\left(\Delta^{\text {lex }}\right)$ for every $i$ and $j$. On the other hand, let $\Delta$ be a simplicial complex on the vertex set $\left\{x_{1}, x_{2}, x_{3}, x_{4}\right\}$ with the facets $\left\{x_{1}, x_{2}\right\}$ and $\left\{x_{3}, x_{4}\right\}$. Then, the facets of $\Delta^{\text {lex }}$ are $\left\{x_{1}\right\},\left\{x_{2}, x_{4}\right\}$ and $\left\{x_{3}, x_{4}\right\}$. Hence, $n_{0}^{1}(\Delta)=4$, while $n_{0}^{1}\left(\Delta^{\text {lex }}\right)=3$. Hence, in general, the inequality $n_{i}^{j}(\Delta) \leq n_{i}^{j}\left(\Delta^{\text {lex }}\right)$ cannot be true if $j \geq 1$. However, we have the following

Corollary. Let $\Delta$ be a simplicial complex and $\Delta^{\text {lex }}$ the lexsegment simplicial complex with the same $f$-vector as $\Delta$. Fix $j \geq 0$ and suppose that

$$
n_{i}^{0}(\Delta)+n_{i}^{1}(\Delta)+\cdots+n_{i}^{j-1}(\Delta)=n_{i}^{0}\left(\Delta^{\text {lex }}\right)+n_{i}^{1}\left(\Delta^{\text {lex }}\right)+\cdots+n_{i}^{j-1}\left(\Delta^{\text {lex }}\right)
$$

for every $i \geq 0$. Then, we have the inequality

$$
n_{i}^{j}(\Delta) \leq n_{i}^{j}\left(\Delta^{\text {lex }}\right)
$$

for every $i \geq 0$. 
Proof. Let $A=K\{\Gamma\} / I_{\Delta}^{\prime}$ and $B=K\{\Gamma\} / I_{\Delta^{\operatorname{lex}}}^{\prime}=K\{\Gamma\} /\left(I_{\Delta}^{\prime}\right)^{\text {lex }}$. Then

$$
\operatorname{dim}_{K}\left(0:_{A} m^{j}\right)_{i}=\sum_{k=0}^{j-1} n_{i-1}^{k}(\Delta)
$$

and

$$
\operatorname{dim}_{K}\left(0:_{B} m^{j}\right)_{i}=\sum_{k=0}^{j-1} n_{i-1}^{k}\left(\Delta^{\text {lex }}\right)
$$

Hence, we can apply (c) of the theorem.

Q. E. D.

Let $\Delta$ be a lexsegment simplicial complex with $f(\Delta)=\left(f_{0}, f_{1}, \ldots\right)$ and $\Delta^{\prime}$ the subcomplex of $\Delta$ obtained by removing all facets of $\Delta$. Then, $\Delta^{\prime}$ is again lexsegment with $f\left(\Delta^{\prime}\right)=\left(\partial_{1}\left(f_{1}\right), \partial_{2}\left(f_{2}\right), \ldots\right)$, and, moreover, the facets of $\Delta^{\prime}$ are just the 1facets of $\Delta$. Hence, it follows that $n_{i-1}^{1}(\Delta)=\partial_{i}\left(f_{i}\right)-\partial_{i}\left(\partial_{i+1}\left(f_{i+1}\right)\right)$ for every $i$. In general, for every $j$ and $i$, we have the formula

$$
n_{i-1}^{j}(\Delta)=\partial_{i}\left(\partial_{i+1}\left(\cdots\left(\partial_{i+j-1}\left(f_{i+j-1}\right)\right) \cdots\right)\right)-\partial_{i}\left(\partial_{i+1}\left(\cdots\left(\partial_{i+j}\left(f_{i+j}\right)\right) \cdots\right)\right) .
$$

\section{REFERENCES}

[A-H-H] A. Aramova, J. Herzog and T. Hibi, Squarefree lexsegment ideals, Math. Z., to appear.

$[\mathrm{B}-\mathrm{H}] \quad$ W. Bruns and J. Herzog, "Cohen-Macaulay Rings," Cambridge University Press, Cambridge / New York / Sydney, 1993. MR 95h:13020

$[\mathrm{B}-\mathrm{H}-\mathrm{V}]$ W. Bruns, J. Herzog and U. Vetter, Syzygies and Walks, in "Commutative Algebra" (G. Valla, et al., Eds.), World Scientific, 1994, pp. 36 - 57.

[C-L] G. F. Clements and B. Lindström, A generalization of a combinatorial theorem of Macaulay, J. Combin. Theory 7 (1969), 230 - 238 MR 40:50

[G-K] C. Greene and D. Kleitman, Proof techniques in the theory of finite sets, in "Studies in Combinatorics" (G.-C. Rota, ed.), Mathematical Association of America, Washington, D.C., 1978, pp. $22-79$. MR 80a:05006

[H] T. Hibi, "Algebraic Combinatorics on Convex Polytopes," Carslaw Publications, Glebe, N.S.W., Australia, 1992.

[Hoc] M. Hochster, Cohen-Macaulay rings, combinatorics, and simplicial complexes, in "Ring Theory II" (B. R. McDonald and R. Morris, eds.), Lect. Notes in Pure and Appl Math., No. 26, Dekker, New York, 1977, pp. $171-223$. MR 58:376

[M-M] M. Möller and F. Mora, New constructive methods in classical ideal theory, J. of Algebra 100 (1986), 138 - 178. MR 88c: 13012

[Sta] R. P. Stanley, "Combinatorics and Commutative Algebra," Birkhäuser, Boston / Basel / Stuttgart, 1983. MR 85b:05002

FB 6 Mathematik und Informatik, Universität-GHS-Essen, 45117 Essen, Germany

E-mail address: mat300@uni-essen.de

Department of Mathematics, Graduate School of Science, Osaka University, ToyONAKA, OSAKA 560, JAPAN

E-mail address: hibi@math.sci.osaka-u.ac.jp 